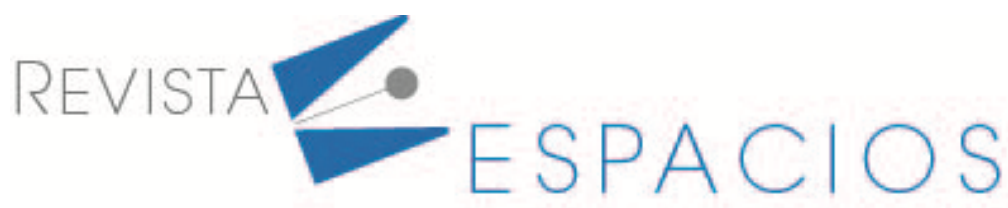

Vol. 42 (02) $2021 \cdot$ Art. 1

\title{
Análisis de la influencia de los mecanismos del gobierno corporativo en la manipulación del resultado contable. Evidencia empírica desde México 2014-2017
}

\section{Analysis of the influence of corporate governance mechanisms on the manipulation of accounting results. Empirical evidence from México 2014-2017}

BARRERA, Viviana ${ }^{1}$

POLO, Sergio D. ${ }^{2}$

\begin{abstract}
Resumen
El estudio analiza la influencia de los mecanismos del Gobierno Corporativo en prácticas de manipulación contable en empresas de los sectores industrial y material de la región norte de México, listadas en la Bolsa Mexicana de Valores durante el periodo 2014-2017, analizándose una muestra formada por 32 Informes Anuales y 32 Cuestionarios del Código Mejores Prácticas Corporativas. El modelo de regresión lineal muestra que la función de auditoría y asamblea de accionistas, disminuyen las prácticas manipuladoras de las cifras contables.

Palabras clave: Mecanismos de Gobierno Corporativo, Gestión de Resultados, Informe financiero, Regresión Lineal.
\end{abstract}

\begin{abstract}
The study analyzes the influence of Corporate Governance mechanisms on accounting manipulation practices in companies in the industrial and material sectors of the northern region of Mexico, listed on the Mexican Stock Exchange during the 2014-2017 period, analyzing a sample consisting of 32 Annual Reports and 32 Questionnaires of the Code of Best Corporate Practices, the linear regression model shows that the audit function and the shareholders' meeting reduce the manipulative practices of the accounting figures.

key words: Corporate Governance Mechanisms, Earnings Management, Financial Report, Linear Regression
\end{abstract}

\section{Introducción}

Una de las problemáticas que ha afectado al sistema bursátil internacional a lo largo de los años, son los escándalos financieros, algunos ejemplos de ello, han sido los casos de las empresas Enron (Estados Unidos, 2001), WorldCom (EUA, 2002), TV Azteca (México, 2003), Parmalat (Italia, 2003), Adecco (Suiza, 2003), Lehman Brothers (Estados Unidos, 2008) y La Polar (Chile, 2011), entre otros, que han afectado la credibilidad de la

\footnotetext{
${ }^{1}$ Docente de la Universidad Autónoma del Estado de Hidalgo, Instituto de Ciencias Económico Administrativas, Área académica de contaduría de la UAEH. vbarreratapia @gmail.com

${ }^{2}$ Profesor Investigador de tiempo completo de la Universidad Autónoma Estado Hidalgo. Instituto de Ciencias Económico Administrativas, Área académica de contaduría de la UAEH. sjimenez221266@yahoo.com.mx
} 
información presentada en los mercados internacionales de capital (Cornejo y Guíñez, 2016). De esta manera, surge la necesidad de mejorar la transparencia corporativa, por lo que, organismos reguladores en los diversos países, han desarrollado marcos normativos para la integración de los reportes financieros del sector empresarial. Por consiguiente, se incorporan principios y prácticas de buen Gobierno Corporativo a las empresas que cotizan en los mercados financieros, con la intención de mejorar la transparencia de sus operaciones y la información generada según el Consejo Coordinador Empresarial (CCE) (2018), creando un ambiente de debates entre los diversos interesados en el tema de prácticas corporativas y su influencia en el manejo de la manipulación contable (García, Gill de Albornoz y Gisbert, 2005).

Por su parte, México en 1999 emite el Código de Mejores Prácticas Corporativas (CMPC) a través del Comité de Mejores Prácticas de Gobierno Corporativo, perteneciente al Consejo Coordinador Empresarial (CCE), cuyo contenido consiste en una serie de principios que a manera de recomendaciones pretendía entre otras cosas, mejorar las funciones del consejo de administración en las empresas y la revelación de información para los stakeholders. El 26 de Octubre del año 2000, la Comisión Nacional Bancaria y de Valores (CNBV), a través de la emisión de la circular 11-33 Disp. XII, hace obligatoria la presentación de información (a mas tardar el 30 de Junio de cada año), relacionada con el Código de Mejores Prácticas Corporativas a las empresas cotizadas en la Bolsa Mexicana de Valores (BMV). Adicionalmente, en las disposiciones de carácter general I, II y XIV se establece como requisito para oferta pública, que las emisoras de valores deban revelar el grado de adhesión al mencionado Código. Para efectos del segundo semestre del 2006, entra en vigor la Nueva Ley del Mercado de Valores, que incorpora importantes disposiciones al tema del Gobierno Corporativo, destacando entre otras, las contenidas en el Capitulo I, art. 12, Sección III art. 19 relativas a la aparición de nuevas figuras societarias y al Capítulo II, Art. 22 relativas a la conversión de las sociedades anónimas públicas en Sociedades Anónimas Bursátiles. La Cámara de Diputados (2015)

Con el objeto de que México pueda cumplir los estándares internacionales recomendados por la Organización para la Cooperación y el Desarrollo Económicos (OCDE) y de la cual forma parte desde el 18 de mayo de 1994, se propone realizar una serie de modificaciones a la Ley General de Sociedades Mercantiles para crear un marco regulatorio eficaz que prevea la implementación por parte de la sociedad anónima de los principios y mejores prácticas de gobierno corporativo (p.2).

Dado lo anterior y con el propósito de mostrar evidencia empírica respecto al efecto que guardan las disposiciones contenidas en la nueva Ley General de Socedades Mercantiles del año 2015, relativas al Gobierno Corporativo en las prácticas de manipulación contable, el presente estudio se orienta en analizar el nivel de influencia que mantienen los mecanismos del Gobierno Corporativo, en las prácticas de manipulación de las cifras contables, considerando para ello un periodo que contempla un año antes de la modificación a la ley (2014), el año 2015 y 2 años posteriores a la disposición legal (2016 y 2017), analizando una muestra formada por empresas mexicanas listadas en la BMV de los sectores industrial y materiales de la región norte de México, en virtud, de la importancia que mantienen estos sectores económicos, considerados una piedra angular para el crecimiento y desarrollo económico del país (Palomino, 2017; Sánchez y Campos, 2010), contribuyendo a la línea de investigación sobre Gobierno Corporativo y Earnings Management y dando evidencia a la acertada propuesta emitida por la CNBV en México. Para alcanzar el objetivo propuesto, el trabajo se estructura en cuatro secciones, después de la introducción se dan a conocer algunos de los estudios de mayor relevancia que se han presentado, en la siguiente sección, se analiza la relación que mantiene el Gobierno Corporativo, con las prácticas de manipulación de los resultados contables, en una tercera parte, se presenta el diseño y metodología de la investigación, el cuarto punto aborda los resultados y conclusiones alcanzados finalizando con las referencias utilizadas. 


\subsection{Revisión de la literatura}

Con el propósito de dar a conocer parte de la literatura previa, que se encarga de estudiar la relación que existe entre la influencia de los mecanismos del Gobierno Corporativo y la propensión que presenta la gerencia en la manipulación de las cifras contables, se muestran en primer lugar, aquellos trabajos relativos al Gobierno Corporativo, posteriormente aquellos centrados en el Earnings Management y concluimos con la mezcla de ambos, es decir, aquellos que explican el condicionamiento que representan las mejores prácticas corporativas en las prácticas manipuladoras de los resultados contables.

\subsection{Gobierno Corporativo}

El Gobierno Corporativo (GC), en sus inicios surgió con la intención de abordar los conflictos derivados por los diferentes intereses entre la administración y los accionistas, lo cual fue ocasionado por la división entre propiedad y control (Jensen y Meckling, 1976). Por lo tanto, el GC, "es una práctica o sistema bajo el cual, las sociedades son dirigidas y controladas" (CCE, 2018. p. 9). El Gobierno Corporativo (GC), tiene como objetivo la creación de confianza a través de la transparencia y rendición de cuentas con la intención de favorecer inversiones a largo plazo, lograr estabilidad financiera e integridad en los negocios como lo establece la Organización para la Cooperación y el Desarrollo Económico (OCDE, 2016). Regularmente el GC, se constituye por la Asamblea General de Accionistas (AA), el Consejo de Administración (CA), el Consejo de Vigilancia (CV) y la Dirección General (DG). La mayoría de los estudios centrados en las prácticas organizacionales, encuentran relaciones significativas entre los mecanismos del Gobierno Corporativo y el performance de las empresas (Garay, González, González y Hernández, 2006; Polo, Hidalgo y Jiménez, 2010; López y Zarza, 2010; Bin y Abbas, 2013; Dedu y Chitan, 2013; Álvarez, 2014; Iwasaki, 2014; Tran, 2014; Macías y Román, 2014; Villeda, 2015; Briano y Saavedra, 2015; Lizarzaburu y del Brío, 2016; Orta y Sierra, 2017; Tejedo y Ferraz, 2018; Jayasuriya, Leon y Yang, 2018; Polo, Villegas, Jiménez y Martín, 2018). El análisis de la literatura previa, muestra también la existencia de estudios, que han desarrollado índices de Gobierno Corporativo, que recogen la información contenida en los códigos de prácticas corporativas y que son considerados como variables predictivas de las cifras contables (Leal y Carvalhal-da-Silva, 2005; Garay et al., 2006; Varshney, Kumar y Vasal, 2012; Jiménez et al., 2014; Javaid y Saboor, 2015; Moreno, Moreno y González, 2018).

\subsection{Manipulación del Resultado/Earnings Management}

El Earnings Management (EM), también conocido como la gestión o manipulación de los resultados contables, se considera como el conjunto de prácticas usadas para alterar la cifra de beneficios reportados en los estados financieros, ya sea para inducir a error a las partes interesadas en el desempeño de la firma o para influir en los resultados contractuales que dependen de las cifras contables (Cornejo y Guíñez, 2016). De esta manera, el cálculo de la variable EM o manipulación contable, se determina a través de la diferencia entre el resultado contable y el flujo de efectivo de las operaciones, denominándose ajustes por devengo, siendo éste, un elemento clave que se descompone en dos secciones, como son la parte discrecional y la no discrecional, los cambios en el componente discrecional representan un intento oportunista (Poveda, 2015). Por lo tanto, para detectar la gestión del resultado o Earnings Management existen diversos modelos, sin embargo, en la literatura no se tiene un consenso sobre cuál de ellos, ofrece el mejor cálculo del nivel de discrecionalidad contable, por lo que cada autor emplea el modelo que considera más apropiado o los modelos que hayan tenido mayor aceptación, entre ellos se encuentran los modelos de Jones (1991), modelo de Jones modificado (1995) y el modelo Jones ajustado al ROA (2005) (Reguera, Laffarga y De Fuentes, 2015). Sin embargo, de acuerdo con Dechow, Sloan y Sweeney (1995), Miko y Kamardin (2014), consideran que el modelo de Jones modificado es el más adecuado para identificar efectivamente la gestión de ganancia, por ello en el presente documento fue el modelo que se utilizó para determinar las acumulaciones discrecionales. 


\subsection{Gobierno Corporativo y Manipulación del Resultado/Earning Management}

Aún en la actualidad, después de casi dos décadas de los escándalos financieros; el Gobierno Corporativo ha ido ganando terreno hasta ocupar un lugar preponderante entre los temas más relevantes para la alta dirección de las organizaciones, tanto para sus estrategias, como para el desarrollo económico y corporativo que la acompañan (Deloitte, 2019). Siendo de gran interés por parte de académicos y profesionales de todo el mundo el desentrañar el impacto que tiene el Gobierno Corporativo y su relación con el Earnings Management en diferentes disciplinas y países (Callao y Jarne, 2011; Malik, Din, Shafi, Butt y Aziz, 2019).

Otros estudios realizados, encuentran una relación significativa entre características intrínsecas organizacionales (resultados contables, tamaño de la junta directiva, número de directivos, retribución al auditor externo, dualidad del CEO, comité de auditoría, la remuneración a directivos, la concentración de la propiedad, el rendimiento de los activos, el costo de capital ) y el nivel de gestión de ganancias (Jamaludin, Sanusi y Kamaluddin, 2015; Latif y Abdullah, 2015; Moss, 2016; Abbadi, Hijazi y Al- Rahahleh, 2016; Essa, Kabir y Nguyen, 2016; Naderi, Mansoori y Alipour, 2017; Chelogoi, 2017; Lawal, Nwanji, Opeyemi y Adama, 2018; Chairina y Kamila, 2018; Muda, Maulana, Siregar y Indra, 2018; Habbash, 2019; Vihi , Abu y Lortima, 2019). La mayoría de los trabajos analizados, muestran evidencia de la influencia del Gobierno Corporativo y de sus mecanismos, en la disminución de la manipulación de los reportes contables, sin embargo, también existen algunos estudios que encuentran un bajo o nulo impacto en el Earnings Management (Kam,2007), sus resultados no muestran una asociación significativa entre la gestión de ganancias y la existencia de comités de auditoría, los resultados obtenidos por el trabajo de Wanjohi (2013), declaran que la gestión de ganancias está relacionada negativamente con la concentración de propiedad y la independencia de la junta, para Lawal, et al. (2018), la independencia del consejo de administración y la independencia de los auditores externos, tienen poco o ningún impacto en la gestión del resultado, similares resultados son obtenidos por Chairina y Kamila (2018), quienes sostienen que la independencia del consejo y de los auditores externos, tienen poco o ningún impacto en la gestión de ganancias, para Muda et al. (2018), cuyas pruebas, indican que la variable de composición del Gobierno Corporativo y el Comité de Auditoría, no tiene ningún efecto en la manipulación de los resultados.

En lo que respecta a México, son escasos los estudios que analizan la influencia que tiene un buen Gobierno Corporativo en la manipulación contable de estados financieros, entre ellos, destacan los trabajos realizados por (Polo, et al., 2018 y Aguilar, Rodríguez y Cortez, 2019). Los estudios realizados, buscan evidencia empírica del grado de cumplimiento de las mejores prácticas de Gobierno Corporativo y el efecto en su rentabilidad, encontrando en sus resultados que las empresas de mayor tamaño son las que más se apegan a las recomendaciones sobre todo en el tamaño del consejo de administración, la función de auditoría y que aquellas empresas que no se apegan a las recomendaciones reflejan menor rentabilidad. Lo que indica un gran acierto, por parte de los organismos reguladores.

De acuerdo a Sáenz y García (2014), quienes analizan si el GC influye en la gestión de ingresos en los mercados latinoamericanos, específicamente en Argentina, Brasil, Chile y México, su estudio les permite afirmar, que cuando un país implementa controles destinados al comportamiento ético, reduce la corrupción, mejora la efectividad del gobierno, este último parece aumentar la calidad y la transparencia de la información financiera emitida por las empresas, mostrando una reducción de las acumulaciones discrecionales.

\section{Metodología}

\subsection{Muestra}

El objetivo del presente estudio, consiste en identificar la influencia de los mecanismos de Gobierno Corporativo en las prácticas de manipulación contable, en las empresas mexicanas de los sectores industrial y material de la 
región norte del país. Es importante mencionar que existen 17 empresas con domicilio corporativo en estados de la zona norte de México, sin embargo, solo se consideran 8 de ellas (que representan el 47.05 por ciento de la población), por ser las que cumplieron con la presentación de los Cuestionarios de Mejores Prácticas Corporativas y los informes anuales correspondientes al periodo comprendido de 2014 al 2017. Finalmente, la muestra queda conformada por 32 cuestionarios del CMPC y 32 informes anuales. El detalle de la muestra se presenta en la Tabla 1.

Tabla 1

Distribución de la muestra por año, tamaño y sector

\begin{tabular}{|c|c|c|c|c|c|c|c|}
\hline \multirow[t]{2}{*}{ Año } & \multicolumn{2}{|c|}{ Materiales } & \multicolumn{2}{|c|}{ Industriales } & \multirow[b]{2}{*}{$\begin{array}{c}\text { Total de } \\
\text { CMPC }\end{array}$} & \multirow[b]{2}{*}{$\begin{array}{c}\text { Total de } \\
\text { Informes } \\
\text { anuales }\end{array}$} & \multirow[b]{2}{*}{ Totales } \\
\hline & CMPC & $\begin{array}{c}\text { Informe } \\
\text { Anual }\end{array}$ & CMPC & $\begin{array}{c}\text { Informe } \\
\text { Anual }\end{array}$ & & & \\
\hline 2014 & 5 & 5 & 3 & 3 & 8 & 8 & 16 \\
\hline 2015 & 5 & 5 & 3 & 3 & 8 & 8 & 16 \\
\hline 2016 & 5 & 5 & 3 & 3 & 8 & 8 & 16 \\
\hline 2017 & 5 & 5 & 3 & 3 & 8 & 8 & 16 \\
\hline Total & 20 & 20 & 12 & 12 & 32 & 32 & 64 \\
\hline
\end{tabular}

Fuente: Elaboración propia

\subsection{Hipótesis}

El estudio de la literatura previa, muestra evidencia de las ventajas que se obtienen por la incorporación de prácticas de buen GC, como son la transparencia y el aumento en la confianza de la información financiera, de esta forma, son diversos los trabajos que muestran evidencia de la efectividad de estos mecanismos corporativos en la disminución de prácticas manipuladoras de los reportes contables realizados por la gerencia (Macías y Román, 2014; Jamaludin, et al., 2015; Ghazali, Shafie y Sanusi, 2015; Abbadi et al., 2016; Lopes, 2018; Nazir y Afza, 2018). La Junta directiva es el órgano más relevante de una sociedad debido a que se encarga de tomar decisiones y llevar el control de la vida de la entidad, así como proteger los derechos e intereses de todos los inversionistas. En este sentido, suponemos que la junta directiva es un factor influyente en la manipulación de los resultados, como lo afirman los trabajos realizados por (Okougbo y Okike, 2015; Jamaludin et al., 2015; Latif y Abdullah, 2015; Abbadi et al., 2016; Essa et al., 2016), de esta manera, se establece la siguiente hipótesis:

$\mathbf{H}_{1}$ : Las prácticas corporativas relativas a la Junta Directiva y Asamblea de Accionistas, mantienen una relación significativa con la manipulación de los resultados contables.

Otra de las variables observadas en el análisis de la literatura, es la relativa al Consejo de Administración, tal como lo afirman los trabajos presentados por (Okougbo y Okike, 2015; Jamaludin et al., 2015; Latif y Abdullah, 2015; Abbadi et al., 2016; Essa et al., 2016), de esta manera se plantea la siguiente hipótesis:

$\mathbf{H}_{2}$ : Los mecanismos de Gobierno Corporativo relativos al Consejo de administración, mantienen una relación significativa con la manipulación de los resultados contables.

El Órgano Intermedio de Auditoría, tiene dentro de sus funciones recomendar al Consejo de Administración los candidatos para auditores externos de la entidad, así como ser el canal de comunicación, revisar reportes de auditorías tanto internas como externas. La auditoría interna, es una herramienta para la función de vigilancia en las operaciones de la sociedad y tener efectividad en los controles internos, siendo una variable representativa en la relación con el Earnings Management (Latif y Abdullah, 2015; Abbadi et al., 2016; Chelogoi, 2017; Chairina y Kamila, 2018), por lo que, se establece la siguiente hipótesis. 
$\mathbf{H}_{3}$ : La Función de Auditoría, mantiene una relación significativa con la manipulación de los resultados contables.

El órgano intermedio de Evaluación y Compensación, en sus diversas funciones sugiere cambios de Director General y altos funcionarios, así como definir el perfil deseado para la contratación, proponer los criterios para su evaluación, compensación y pagos por separación, analizar el Código de Ética, el sistema de información de hechos indebidos y protección de los informantes. Este mecanismo ha sido analizado por diversos investigadores entre los cuales encontramos el de Brick, Palmon y Wald, 2005 que encontró evidencia de una asociación entre el exceso de compensación de directores y gerentes con un bajo rendimiento de la empresa, mientras que Dah y Frye, 2017 encontraron que los directores sobre compensados exacerban los problemas de agencia, conducen a menor sensibilidad de rotación del CEO, disminución de sensibilidad de pago por desempeño del CEO (Brick, Palmon y Wald, 2005), por ello se puede vincular a una estructura débil de GC, mientras que un GC fuerte evita la manipulación en las ganancias (Abbadi et al., 2016; Nazir y Afza, 2018; Saenz y García, 2014), por lo que se establece la siguiente hipótesis.

$\mathrm{H}_{4}$ : La Función de Evaluación y Compensación, mantiene una relación significativa con la manipulación de los resultados contables.

Las mejores prácticas de GC, dan lugar a unas finanzas transparentes que generan confianza a los accionistas e inversionistas en la toma de decisiones (Galaz y Ruiz, 2012), en específico el órgano intermedio de finanzas y planeación, tiene como función definir las estrategias a seguir para lograr estabilidad y permanencia en el tiempo, siendo elemento básico para la conservación del patrimonio de los inversionistas, además de proporcionar un crecimiento sostenido, mayor competitividad y guía en el diagnóstico de la salud financiera de la empresa, entre sus principales objetivos, se tiene garantizar la disponibilidad de recursos financieros requeridos, mecanismo para su uso adecuado, prevención de fraude y corrupción (González, 2014). Así también afirman Macías y Román (2014), López, García y Rodríguez (2006), que a mayor adhesión al código y disposiciones legales mejora el desempeño operativo, por lo que se establece la siguiente hipótesis.

$\mathbf{H}_{5}$ : La Función de Finanzas y Planeación, mantiene una relación significativa con la manipulación de los resultados contables.

\subsection{Medición de variables}

\subsubsection{Variable Dependiente: Gestión del Resultado/Earnings Management}

Son variados los trabajos, que han utilizado diferentes metodologías para el cálculo de la variable predictiva de la discrecionalidad gerencial (Ghazali, Shafie y Sanusi, 2015; Jamaludin et al., 2015; Nazir y Afza, 2018), siendo el más utilizado el modelo de Jones modificado por Dechow en 1995. (Shah, Zafar y Durrani, 2009; Saenz y García, 2014; Ismail, Zakaria y Sata, 2015; Abbadi et al., 2016; Lopes, 2018; Alareeni, 2018). Para efectos del presente estudio, la manipulación de las cifras contables, se calcula con el componente discrecional de los ajustes, propuesto por Dechow (1995), que obtiene los ajustes discrecionales y los no discrecionales, a través, del ajuste por devengo total y cuya ecuación es la siguiente:

$$
A D T=(\triangle A C-\Delta \text { Tesorería })-(\triangle P C-\triangle D e u d a c p)-D e p
$$

En donde:

$A D T=$ Ajuste por devengo total

$\triangle \mathrm{AC}=$ Variación de activo circulante

$\triangle$ Tesorería $=$ Variación en el cashflow

$\triangle \mathrm{PC}=$ Variación en pasivo circulante

$\triangle$ Deudacp $=$ Variación en la deuda con vencimiento a corto plazo

Dep = Amortización y depreciación del ejercicio 
Una vez determinada la variable que explica los ajustes por devengo totales (ADT), Dechow (1995), propone la estimación del componente discrecional de los ajustes de devengo, regresando los ajustes de devengo observados, sobre el cambio en ventas ( $\Delta$ Ventas) y el valor bruto del inmovilizado (Inmov).

$$
\frac{A D T_{i t}}{A i^{t-1}}=\hat{\beta} 0 \frac{1}{A i^{t-1}}+\hat{\beta} 1 \frac{\Delta \text { Ventas }_{i t}}{A i^{t-1}}+\hat{\beta} 2 \frac{\text { Inmov }_{i t}}{A i^{t-1}}+\epsilon_{\mathrm{it}}
$$

En donde:

Los subíndices i y $t$, hacen referencia a cada empresa y año, respectivamente.

$\mathrm{Ai}=$ Activos Totales

$\Delta$ Ventas $=$ Cambio en ventas

Inmov = Material inmovilizado (activo fijo)

A partir de las estimaciones de los coeficientes $\left(b^{\wedge} 0, b^{\wedge} 1, b^{\wedge} 2\right)$, se calcula el componente no discrecional de los ajustes de devengo (ADND), para cada empresa de la muestra, utilizando la versión modificada del modelo de Jones (1991), propuesta por Dechow (1995), donde se disminuye la partida contable referente a las cuentas por cobrar en el cambio en ventas.

$$
A D N D=B_{0} 1 / A_{i t-1}+B_{1} \Delta \text { Sales }_{i t}-\Delta C C_{i t} / A_{i t-1}+B_{2} \text { Inmov }_{i t} / A_{i t-1}
$$

En donde:

Los subíndices i y $t$, hacen referencia a cada empresa y año respectivamente.

ADND it = Ajuste por devengo no discrecional.

$\triangle C C=$ Cambio en cuentas por cobrar.

Inmov= Material inmovilizado (activo fijo)

Se define el componente discrecional de los ajustes de devengo (ADD), para la empresa i en el año t, como la diferencia entre los ajustes de devengo totales observados y los ajustes de devengo no discrecionales.

$$
A D D_{i t}=A D T_{i t}-A D N D_{i t}
$$

En donde:

ADDit $=$ Ajustes de devengo discrecionales.

ADTit = Ajustes de devengo totales.

ADNDit $=$ Ajustes de devengo no discrecionales

\subsubsection{Variable independiente: Gobierno Corporativo}

Para evaluar el efecto del Gobierno Corporativo en los ajustes de devengo discrecionales (Earnings Management) y dado que los Códigos de Mejores Prácticas Corporativas, contienen dimensiones (Asamblea de Accionistas, Consejo de Administración, Función de Auditoría, Evaluación y Compensación y Finanzas y Planeación), se calculan índices por dimensión similares a los propuestos por Leal y Carvahal, 2005; Garay et al., 2006; Varshney et al., 2012; Jiménez et al., 2014; Javaid y Saboor, 2015 y Moreno, Moreno y González 2018, cuya expresión es la siguiente:

$$
\text { F_Index }(x)=\sum R A+/\left(\sum R A+\right)+\left(\sum R N-\right)
$$

Donde:

F_Index = Es el índice de Gobierno Corporativo por dimensión

$\overline{R A}=$ Son las respuestas afirmativas, que refieren a aspectos del código aplicados por las empresas

$\mathrm{RN}=$ Son las respuestas negativas, que refieren a aspectos del código no aplicados por las empresas 


\subsubsection{Variables de control}

Otros factores distintos de las características del Gobierno Corporativo que también contribuyen en la práctica de gestión de ganancias y que han sido utilizados en estudios anteriores, como lo demuestran los trabajos de (Ismail et al., 2015; Abbadi et al., 2016 y Alareeni, 2018) sobre este fenómeno, se encuentra el tamaño de la empresa que se ha medido a través del logaritmo natural de los activo totales al final de año, el nivel de apalancamiento, medido a través de la relación entre deuda y activos totales, considerando que por tener mayor deuda, se tiene mayor probabilidad de la manipulación contable. De acuerdo con Abbadi et al. (2016), incluyen otros dos indicadores sobre el rendimiento de la empresa, tal como el crecimiento de las ventas y rendimiento de los activos, en el cual, el primero se mide con la diferencia de las ventas del año, con las del periodo anterior, esperando que las empresas que tienen un mayor crecimiento en las ventas, tienen menor motivación para la manipulación contable.

\subsubsection{Modelo empírico}

Atendiendo la literatura previa y con la intención de conocer si los mecanismos del Gobierno Corporativo, influyen en la disminución del Earnings Management, se calcula el valor absoluto de la variable manipuladora de las cifras contables y de manera similar a los trabajos realizados por Ali, Butt y Hasan (2009); Abbadi et al., (2016); Lawal et al., (2018); Aguilar et al., (2019) entre otros, se presenta el siguiente modelo de regresión lineal:

\section{$A D D \_A B S=b_{0}+b_{1}(S P)+b_{2}(R O A)+b_{3}(T a m a \tilde{o})+b_{4}\left(I \_E C\right)+b_{5}\left(I \_F A\right)+b_{6}\left(I \_A A\right)+b_{7}\left(I \_C A\right)+b_{8}\left(I \_P F\right)+e$}

\section{Donde:}

ADD_ABS = Representa el valor absoluto de las acumulaciones discrecionales

$\mathrm{SP}=$ Sector de pertenencia de las empresas (Tomado de la clasificación que realiza la BMV)

ROA = Rentabilidad Económica

Tamaño = Tamaño de la empresa (Total de activos al 31 de diciembre de cada año)

I_FA = Índice de la Función de Auditoría

I_EC= Índice de Evaluación y Compensación

I_AA= Índice de Asamblea de Accionistas

I_CA= Índice de Consejo de Administración

I_PF= Índice de Planeación y Finanzas

\section{Resultados}

\subsection{Análisis descriptivos}

El comportamiento de las variables GC y EM, respecto a las empresas dedicadas al sector de los materiales, se presenta en la Figura 1, donde la tendencia de las prácticas que manipulan la información financiera, es negativa y aunque la variable predictora del Gobierno Corporativo también lo es, estas prácticas societarias, disminuyen significativamente el Earnings Management en los dos últimos años.

Figura 1

Comportamiento de los índices de GC y EM por año en el sector de materiales

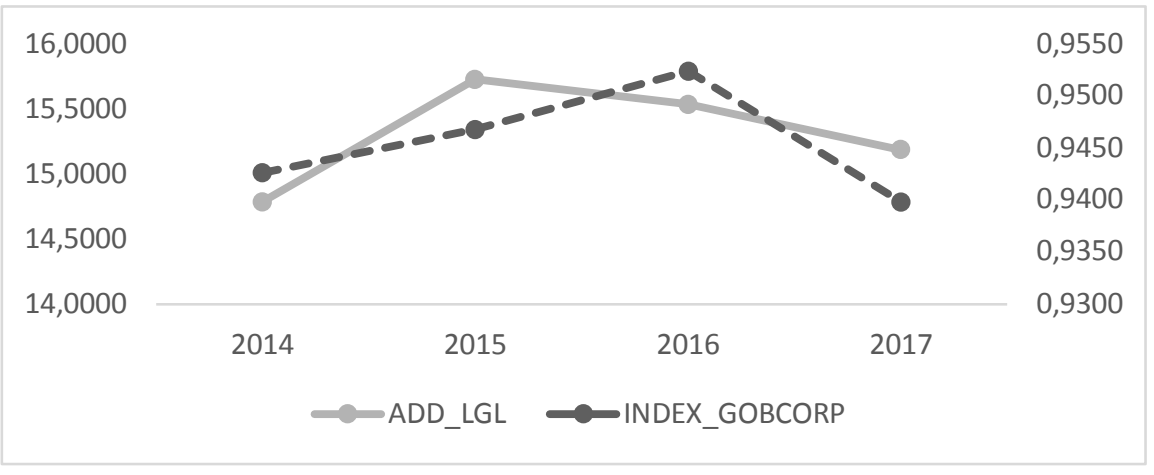

Fuente: Elaboración Propia 
La Figura 2, muestra las compañías dedicadas al sector industrial, en donde el comportamiento de las variables analizadas, tal como se esperaba, a mayor adhesión a las recomendaciones corporativas realizadas por los organismos reguladores mexicanos, menor seria la propensión a gestionar los resultados financieros, tendencia con mayor impacto en el último año del estudio. Los resultados presentados, confirman el acierto de la Comisión Nacional Bancaria y de Valores en México, respecto al fortalecimiento de la confianza de los inversores en el Mercado Bursátil local, a través de un sólido Gobierno Corporativo y un bajo nivel de manipulación contable.

Figura 2

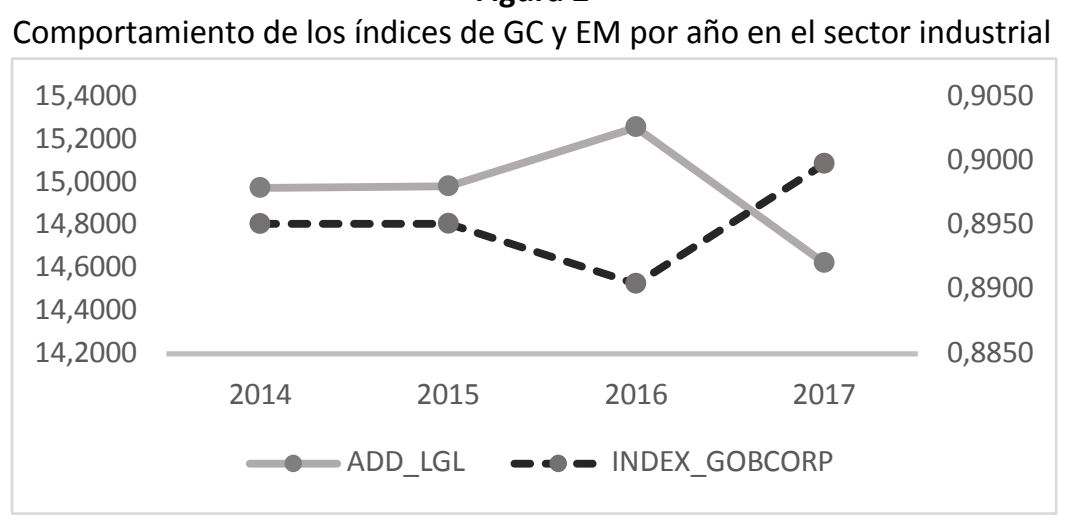

Fuente: Elaboración propia

\subsection{Técnicas de análisis}

Con el propósito de contrastar las hipótesis planteadas y conociendo que la distribución de los datos es normal (Test de Shapiro W., significancia de .918 >.05 por lo que, estadísticamente, se considera que los datos provienen de una distribución normal), se procede a la utilización de pruebas paramétricas o multivariantes (modelos con mayor potencia, que sus homólogos no paramétricos, (Gómez-Biedma, Vivó y Soria, 2001), específicamente a través, de modelos de regresión lineal, que permiten conocer la relación existente entre las variables utilizadas. Para conocer, si la varianza de los errores, no es constante en todas las observaciones realizadas (Heterocedasticidad), se aplica el Test de White, con el programa Stata, en donde el valor de $p=.4167>0.05 \mathrm{y}$ por tanto, se considera que existe homocedasticidad.

El factor inflacionario de la varianza (FIV), permite señalar el nivel de multicolinealidad en las variables del modelo, donde a mayor valor del FIV, mayor problema o colinealidad tiene la variable, como regla práctica, se tiene que si el FIV de una variable, es superior a 10, se dice que la misma, presenta multicolinealidad (Gujarati y Porter, 2010), por ello, en nuestro caso, los valores del VIF de las variables, oscilan de 1.052 a 2.039, por lo que, se puede sostener que el modelo de regresión lineal, no presenta problemas de multicolinealidad en sus predictivas. Por último, para detectar la correlación serial, se utiliza la prueba de Durbin Watson, que obtiene un valor de 2.515 y dado que este se ubica cercano a 2, se considera que no existe autocorrelación en el modelo.

\subsection{Resultados del modelo}

Son variables relevantes del modelo de regresión lineal (Tabla 2), el tamaño de la empresa (medido por el total de sus activos, al 31 de diciembre de cada año), el Índice de la Función de Auditoría, Índice de Evaluación y Compensación e Índice de Asamblea de Accionistas. En este sentido, podríamos referir, que los mecanismos corporativos, relativos a la Función de Auditoría y el Índice de Asamblea de Accionistas, disminuyen sustancialmente la incidencia de prácticas manipuladoras de la información financiera, de las empresas analizadas (al 95\% de confianza, en ambos casos), pues el signo negativo de su coeficiente, disminuye el valor de los ajustes por devengo discrecionales realizados por la gerencia y por tanto, menor es el Earnings Management, estos resultados son consistentes con los presentados, entre otros, por (Ali, Butt y Hassan, 2009; Ismail et al., 
2015; Abbadi, et al., 2016 y Lawal et al., 2018) así mismo, dan evidencia de la efectividad del Gobierno Corporativo en la disminución de la gestión de los resultados contables, reportados por las empresas de la muestra. En tal caso, aceptamos la $H_{1}$ : Las prácticas corporativas relativas a la Junta Directiva y Asamblea de Accionistas, mantienen una relación significativa con la manipulación de los resultados contables y $H_{3}$ : La Función de Auditoría, influye significativamente en la manipulación de los resultados contables. Por último, el modelo estadístico de regresión predice la manipulación de la información financiera en un $87.8 \%$, tal y como lo demuestra su $R^{2}$ ajustado. Respecto a las características empresariales, los resultados muestran, que la variable tamaño de la compañía (medido por su total de activos al 31 de diciembre de cada año), mantiene una relación estadísticamente significativa (al 99\% de confianza), con las prácticas manipuladoras de las cifras contables de tipo positivo, lo que nos permitiría afirmar, que las empresas con mayor tamaño, son aquellas que manipulan en mayor medida la información financiera, esto puede ser debido, al número de operaciones que estas compañías desarrollan y por tanto, más grande es el volumen de ajustes que realizan. El resultado es consistente con los trabajos realizados por (Nalarreason, Sutrisno y Mardiati, 2019; Asim e Ismail, 2019). Por último, la variable relativa a la evaluación y compensación, muestra una relación significativa con la manipulación de los resultados a un $99 \%$ de confianza sin embargo, la relación es positiva y por tanto, no es limitativa del Earnings Management, aceptando $\mathrm{H}_{4}$ : La Función de Evaluación y Compensación, mantiene una relación significativa con la manipulación de los resultados contables. No son variables relevantes del modelo, las relativas a la Razón financiera de Deuda, la Rentabilidad Económica y el Índice de Consejo de Administración.

Tabla 2

Modelo de Regresión Lineal de los Ajustes por Devengo Discrecionales

\begin{tabular}{|c|c|c|c|c|c|c|}
\hline \multicolumn{7}{|c|}{$\begin{array}{c}A D D \_A B S=\beta_{0}+\beta_{1}(S P)+\beta_{2}(A \tilde{n o})+\beta_{3}(R O A)+\beta_{4}(\text { Tamaño })+\beta_{5}\left(I \_E C\right)+\beta_{6}\left(I \_F A\right)+\beta_{7}\left(I \_A A\right)+\beta_{8}\left(I \_C A\right)+ \\
\beta_{9}\left(I \_P F\right)+e\end{array}$} \\
\hline \multirow[t]{2}{*}{ Variables } & \multicolumn{2}{|c|}{$\begin{array}{l}\text { Coeficientes no } \\
\text { estandarizados }\end{array}$} & \multirow{2}{*}{$\begin{array}{c}\text { Coeficientes } \\
\text { estandarizados } \\
\text { Beta }\end{array}$} & \multirow[t]{2}{*}{$\mathrm{T}$} & \multirow[t]{2}{*}{ Sig. } & \multirow{2}{*}{$\begin{array}{c}\begin{array}{c}\text { Estadísticas } \\
\text { de } \\
\text { colinealidad }\end{array} \\
\text { VIF }\end{array}$} \\
\hline & B & $\begin{array}{l}\text { Desv. } \\
\text { Error }\end{array}$ & & & & \\
\hline (Constante) & 13177 & 38011 & & 3.467 & .002 & \\
\hline TAMAÑO & .129 & .012 & .848 & $\begin{array}{l}10.44 \\
7\end{array}$ & .000 & 1.296 \\
\hline $\begin{array}{l}\text { INDICE DE FUNCION DE } \\
\text { AUDITORIA }\end{array}$ & -54255 & 24262 & -.204 & -2.236 & .035 & 1.645 \\
\hline RAZÓN DE DEUDA (PT/AT) & -54279 & 77671 & -.086 & -.699 & .491 & 2.979 \\
\hline $\begin{array}{l}\text { RENTABILIDAD } \\
\text { ECONÓMICA (ROE) }\end{array}$ & -46661 & 16525 & -.026 & -.282 & .780 & 1.732 \\
\hline $\begin{array}{l}\text { INDICE DE CONSEJO DE } \\
\text { ADMINISTRACION }\end{array}$ & -38925 & 16671 & -.026 & -.233 & .817 & 2.354 \\
\hline $\begin{array}{l}\text { INDICE DE EVALUACION Y } \\
\text { COMPENSACION }\end{array}$ & 79683 & 19147 & .601 & 4.162 & .000 & 4.100 \\
\hline $\begin{array}{l}\text { INDICE DE ASAMBLEA DE } \\
\text { ACCIONISTAS }\end{array}$ & -14945 & 41608 & -.502 & -3.592 & .001 & 3.844 \\
\hline *** Significatividad 99\%; & Signif & $\begin{array}{l}\mathrm{R} 2 \\
\text { Ano } \\
\text { idad } 95 \\
\mathrm{AL}\end{array}$ & $\begin{array}{l}=0.878 \\
=0.000 \\
* \text { Significativid } \\
\text { _ABS }\end{array}$ & $0 \%$ a. & ble & ndiente: \\
\hline
\end{tabular}

a Variable dependiente: ADD_ABS 


\section{Conclusiones}

Los resultados del análisis descriptivo, referente al comportamiento de las variables Gobierno Corporativo y las prácticas manipuladoras de la información financiera del 2014 al 2017, nos permiten concluir que las modificaciones realizadas a la Ley de Sociedades Mercantiles en 2015, están aumentando la calidad de las cifras contables, a través de la implementación de las recomendaciones contenidas en los Códigos de Mejores Prácticas Corporativas, pues a pesar de que la adhesión a estas recomendaciones no se cumplen al 100 por ciento, la tendencia de las actividades de gestión de las cifras, disminuye con mayor impacto en los dos últimos años, lo que representa, la generación de confianza en el público inversor, apoyando el crecimiento de la economía mexicana. Por último, el análisis multivariante, da evidencia de la importancia de la Función de Auditoría y Junta Directiva y Asamblea de Accionistas, en las organizaciones estudiadas y cómo estas actividades de control y gobierno, actúan como limitantes de las prácticas manipuladoras de resultados.

\section{Referencias bibliográficas}

Abbadi, S. S., Hijazi, Q. F. y Al-Rahahleh, A. S. (2016). Corporate governance quality and earnings management: Evidence from Jordan. Australasian Accounting, Business and Finance Journal, 10(2), 54-75. https://doi.org/10.14453/aabfj.v10i2.4

Aguilar, I. A., Rodríguez, M.P. y Cortez, A. (2019). ¿Son las prácticas de Gobierno Corporativo y de earnings management relevantes para el valor de las empresas en México? Contaduría y Administración, 64(4), 120. Recuperado de https://www.researchgate.net/publication/343756574_Son_las_practicas_de_gobierno_corporativo_y_d e_earnings_management_relevantes_para_el_valor_de_las_empresas_en

Alareeni, B. (2018). Does corporate governance influence earnings management in listed companies in Bahrain Bourse? Journal of Asia Business Studies, 12(4), 551-570. https://doi.org/10.1108/JABS-06-2017-0082

Ali, S., Butt S.A. y Hasan, A. (2009). Corporate Governance and Earnings Management an Empirical Evidence Form Pakistani Listed Companies. European Journal of Scientific Research, 26(4), 624-638. Recuperado de https://ssrn.com/abstract=1732510

Álvarez, A. (2014). Un estudio del Gobierno Corporativo: SACYR, ACCIONA y ACS. Repositorio, Universidad de León, 1-88.

Recuperado de http://hdl.handle.net/10612/3788

Asim, A. y Ismail, A. (2019). Impact of Leverage on Earning Management: Empirical Evidence from the Manufacturing Sector of Pakistan. Journal of Finance and Accounting Research, 1(1), 70-91. https://ojs.umt.edu.pk/index.php/jfar/article/view/17

Bin, Y. y Abbas, Z. (2013). Compliance and Multidimensional Firm Performance: Evaluating the Efficacy of RuleBased Code of Corporate Governance. Economic Modelling, 35(2), 565-575. Recuperado de https://doi.org/10.1016/j.econmod.2013.08.015

Briano, T. y Saavedra, M. L. (2015). La composición del consejo de administración y la estructura accionaria como factores explicativos de la transparencia en el Gobierno Corporativo en Latinoamérica: Evidencia en empresas cotizadas de Argentina, Brasil, Chile y México. Estudios Gerenciales, 31(136), 275-286. https://doi.org/10.1016/j.estger.2015.02.001 
Brick, I. E., Palmon, O. y Wald, J. K. (2005). CEO compensation, director compensation, and firm performance: Evidence of cronyism?. Journal of Corporate Finance, 12(3), 403-423. Recuperado de https://www.sciencedirect.com/science/article/pii/S0929119905000738

Cámara de Diputados, (2015). Gaceta Parlamentaria. Recuperado de http://gaceta.diputados.gob.mx/PDF/62/2015/mar/20150318-III.pdf

Callao, S. y Jarne, J.I. (2011). El Impacto de la Crisis en la Manipulación Contable. Revista de Contabilidad, 14(2), 59-85. https://doi.org/10.1016/S1138-4891(11)70028-0

Chairina, A. y Kamila, Z. (2018). The Effect of Good Corporate Governance and Earnings Management to Corporate Social Responsibility Disclosure. Academy of Accounting and Financial Studies Journal, 22(1), 116. Recuperado de https://www.abacademies.org/articles/the-effect-of-good-corporate-governance-andearnings-management-1528-2635-22-1-114.pdf

Chelogoi, S. K. (2017). Effect of corporate governance on earnings management of firms listed in nairobi securities exchange. International Journal of Economics, Commerce and Management, 641-654.

Consejo Coordinador Empresarial (CCE) (2018). Código de Principios y Mejores Prácticas de Gobierno Corporativo. Recuperado de https://www.cce.org.mx/comite-de-mejores-practicas-de-gobiernocorporativo/

Cornejo, E. y Guíñez, N. (2016). Motivaciones de la Gerencia para Manipular la Cifra de Beneficio Contable. Revista Academia de Negocios, 2(1), 69-84.

https://www.researchgate.net/publication/304628905

Dah, M.A. y Frye, M.B. (2017). Is board compensation excessive. Journal of Corporate Finance, 45, 566-585. https://doi.org/10.1016/j.jcorpfin.2017.06.001

Dechow, P.M., Sloan, R.G. y Sweeney, A.P. (1995): “Detecting earnings management", The Accounting Review, LXX, (2), pp. 193-225. Recuperado de https://www.jstor.org/stable/248303?seq=1

Dedu, V. y Chitan, G. (2013). The influence of internal corporate governance on bank performance-An empirical analysis for Romania. Elsevier, 99, 1114-1123. https://doi.org/10.1016/j.sbspro.2013.10.585

Deloitte. (2019), 60 Estudio de Mejores Prácticas en Gobierno Corporativo. Retrieved fromhttps://www2.deloitte.com/content/dam/Deloitte/mx/Documents/risk/2018/6to-Estudio-MejoresPracticas-Gobierno-Corporativo.pdf

Essa, S., Kabir, R. y Nguyen, H. T. (2016). Does Corporate Governance Affect Earnings Management? Evidence from Vietnam?. Paper presented at 2016 Vietnam symposium in Banking and Finance (VSBF), Hanoi, Viet Nam. Recuperado de https://www.semanticscholar.org/paper/Does-Corporate-Governance-AffectEarnings-Evidence-Essa-Kabir/94c87da76711e8e27475b8dc794fad0174eb2383?p2df

Galaz, Y. y Ruiz, U. (2012). Mejores prácticas enfocadas a las finanzas Recuperado de https://www2.deloitte.com/content/dam/Deloitte/mx/Documents/risk/Gobierno-Corporativo/mejorespracticas-enfocadas-finanzas.pdf

Garay, U., González, G., González, M. y Hernández, Y. (2006). Índice de buen Gobierno Corporativo y desempeño financiero en la Bolsa de Valores de Caracas. Estudio IESA, 24, 4-21. 
García, B., Gill de Albornoz, B. y Gisbert, B. (2005). La investigación sobre "Earnings Managements". Revista Española de Financiación y Contabilidad, XXXIV(127), 1001-1033.

https://doi.org/10.1080/02102412.2005.10779570

Gaytán C.J., Sánchez, J. Vizcaíno A,J. y Vargas J.A. (2016) La competitividad y su relación con la gestión del conocimiento y el mercado global. Recuperado de http://cucea.udg.mx/sites/default/files/documentos/adjuntos_pagina/competitividad_y_su_relacion_con _la_gestion_del_conocimiento_y_mercado_global.pdf

Ghazali, A., Shafie, N. y Sanusi, Z. (2015). Earnings Management: An Analysis of Opportunistic Behaviour, Monitoring Mechanism and Financial Distress. Procedia Economics and Finance, 28(April), 190-201. https://doi.org/10.1016/s2212-5671(15)01100-4

González, M. (2014). Función de Finanzas y Planeación. Recuperado de http://contaduriapublica.org.mx/2014/10/06/funcion-de-finanzas-y-planeacion/

Gómez-Biedma, S. y Vivó, M., Soria, E., (2001). Pruebas de significación en Bioestadística. Revista de diagnóstico Biológico, 50(4).207-218. Recuperado en 17 de abril de 2020, de http://scielo.isciii.es/scielo.php?script=sci_arttext\&pid=S0034-79732001000400008

Gujarati, D. y Porter, D. (2010). Flexibilización de los supuestos del modelo clásico. En Econometría. (pp. 327329). México, D.F: MC Graw Hill

Habbash, M. (2019), "The Role of Corporate Governance Regulations in Constraining Earnings Management Practice in Saudi Arabia", Research in Corporate and Shari'ah Governance in the Muslim World: Theory and Practice, Emerald Publishing pp. 127-140. https://doi.org/10.1108/978-1-78973-007-420191011

Ismail, N. I., Zakaria, N. B. y Sata, F. H. A. (2015). Auditors Roles Towards the Practice of Earnings Manipulation among the Malaysian Public Firms. Procedia Economics and Finance, 28(April), 145-150. https://doi.org/10.1016/s2212-5671(15)01093-x

Iwasaki, I. (2014). Global Financial Crisis Corporate Governance and Firm Survival: The Russian Experience. Journal of Financial Economics, 42(1), 178-211. https://doi.org/10.1016/j.jce.2013.03.015

Jamaludin, N. D., Sanusi, Z. M., y Kamaluddin, A. (2015). Board Structure and Earnings Management in Malaysian Government Linked Companies. Procedia Economics and Finance, 28(December), 235-242. https://doi.org/10.1016/s2212-5671(15)01105-3

Javaid, F. y Saboor, A. (2015). Impact of Corporate Governance index on Firm Performance: Evidence from Pakistani manufacturing sector. Journal of Public Administration and Governance, 4(3), 1. https://doi.org/10.5296/jpag.v5i2.7498

Jayasuriya, F., Leon, L., y Yang, H. (2018). Corporate governance and default Prediction: reality test. Applied Economics, DOI:10.1080/00036846.2018.1558351

Jensen, M. y Meckling, W. (1976). Theory of The Firm Manajerial Behaviour, Ageny Cost and Ownership Structure. Journal of Financial Economics, 3, 305-360. https://doi.org/10.1016/0304-405X(76)90026-X

Jiménez, S.D.P., Uribe, H.M., Arteaga, G.M. y Moreno, E.S.V. (2014). “Análisis del Cumplimiento del Código de Mejores Prácticas Corporativas en México". Boletín Científico de las Ciencias Económico Administrativas del ICEA, 3(5). Recuperado de https://www.uaeh.edu.mx/scige/boletin/icea/n5/e10.html 
Jones, J. J. (1991). Earnings Management During Import Relief Investigations. Journal of Accounting Research, 29(2), 1993-228. https://doi.org/10.2307/2491047

Kam, P. M. (2007). Corporate Governance and Earnings Management: Some Evidence from Hong Kong Listed Companies (Doctor of Philosophy), University of the Sunshine Coast Maroochydore DC Queensland 4558 Australia. http://research.usc.edu.au/vital/access/manager/Repository/usc:1511

Latif, A.S. y Abdullah, F. (2015). The Effectiveness of Corporate Governance in Constraining Earnings Management in Pakistan. The Lahore Journal of Economics, 20(1), 135-155. https://doi.org/DOI: 10.35536/lje.2015.v20.i1.a5

Lawal, A.I., Nwanji, T.I., Opeyemi, O.O. y Adama, I.J. (2018). Can corporate governance mechanisms deter earnings management? Evidence from firms listed on the Nigerian Stock Exchange, the IEB international journal of finance, 17, 220-233. https://dialnet.unirioja.es/servlet/articulo?codigo=6639139

Leal, R. y. Carvalhal-da-Silva, A. (2005). Corporate Governance and Value in Brazil (and in Chile). National Scientific and Technological Development Council of Brazil, 77. http://dx.doi.org/10.2139/ssrn.726261

Lizarzaburu, E. y Del Brío, J. (2016). Responsabilidad Social Corporativa y Reputación Corporativa en el sector financiero de países en desarrollo. Georgetown University, 10(1), 42-65. https://doi.org/10.3232/GCG.2016.V10.N1.02

Lopes, A. P. (2018). Audit Quality and Earnings Management: Evidence from Portugal. Athens Journal of Business \& Economics, 4(2), 179-192. https://doi.org/10.30958/ajbe.4.2.4

López, F. y Zarza, C. (2010). Gobierno Corporativo y factores determinantes del cambio de firma auditora en la gran empresa española. Revista Española de Financiación y Contabilidad, XXXIX(147), 521-549. Recuperado de https://dialnet.unirioja.es/servlet/articulo?codigo=3317142

López, M.V., García, A. y Rodríguez, L. (2006). Gobierno Corporativo y desempeño financiero Un estudio empírico. dialnet, 37, 31-43. Recuperado de https://dialnet.unirioja.es/servlet/articulo?codigo=4162460

Macías, A., y Román, F. (2014). Consecuencias Económicas de la Reforma de Gobierno Corporativo en un Mercado de Capitales Emergentes. El trimestre económico, LXXXI(2), 357-412. Recuperado de http://www.scielo.org.mx/scielo.php?pid=S2448-718X2014000200357\&script=sci_abstract\&tIng=es

Malik, A., Din, S.U., Shafi, K., Butt, B.Z. y Aziz, H. (2019). Earning Management and the Likelihood of Financial Distress in Banks. Public Finance Quarterly, 64(2), 208-221.

Miko, N.U., y Kamardin, H. (2014). Detecting earnings management: Comparative analysis of models in Nigeria. Journal of Accounting Frontier, 16(2), 68-76. Recuperado de https://www.researchgate.net/profile/nuraddeen_miko/publication/311743143_detecting_earnings_man agement_comparative_analysis_of_models_in_nigeria/

Moreno, H., Moreno, P.H., y González, G. (2018). El Impacto de las Buenas Prácticas en las Empresas que cotizan en La Bolsa Mexicana de Valores Gobierno Corporativo. Revista Internacional La Nueva Gestión Organizacional, (9), 38-60. Recuperado de https://uatx.mx/publicaciones/revistas/fcea/RI0712201809.pdf\#page=41

Moss, S. S. (2016). The Impact of corporate governance and earning management practices on cost of equity capital: Evidence from thai listed companies. ACRN Oxford Journal of Finance and Risk Perspectives, 160- 
182. Recuperado de https://www.semanticscholar.org/paper/THE-IMPACT-OF-CORPORATEGOVERNANCE-AND-EARNINGS-ON-MOSS/22c78d87507c3d03f9dd1aa38bac3278fcab929c

Muda, I., Maulana, W., Siregar, H.S., y Indra, N. (2018). The Analysis of Effects of Good Corporate Governance on Earnings Management in Indonesia with Panel Data Approach. Iran. Econ. Rev, 22(2), 599-625. https://dx.doi.org/10.22059/ier.2018.66169

Naderi, S., Mansoori, E. y Alipour, O. (2017). The Impact of Corporate Governance on Earnings Management for Listed Companies in Tehran Stock Exchange. International Academic Journal of Accounting and Financial Management , ,2(1), 98-106 Recuperado de https://www.iaiest.com/datacms/articles/20191102084705amIAJAFM1510010.pdf

Nalarreason, K.M., Sutrisno T, y Mardiati, E.,(2019). Impact of Leverage and Firm Size on Earnings Management in Indonesia. Volume 6, Issue 1, 2019 Pages: 19-24

Nazir, M. y Afza T. (2018). Impact of Corporate Governance on Discretionary Earnings Management a Case of Pakistani Firms. Pakistan Economic and social Review, 56(1), 157-184. Recuperado de http://pu.edu.pk/images/journal/pesr/PDF-FILES/7-v56_1_18.pdf

Okougbo, P. y Okike, E. (2015). Corporate Governance and Earnings Management: Empirical Evidence from Nigeria. Corporate Ownership and Control, 12(4), 312-326. Recuperado de https://www.researchgate.net/publication/305954881

Orta, M., y Sierra. L. (2017). Efectividad de los Mecanismos de Gobierno Corporativo: Un Estudio Empírico. Revista Española de Financiación y Contabilidad, 39, 1-30. Recuperado de https://docplayer.es/19028491Efectividad-de-los-mecanismos-de-gobierno-corporativo-un-estudio-empirico.html

Palomino, M. (2017). Importancia del sector industrial en el desarrollo económico: Una revisión al estado del arte. Revista Estudios de Políticas Públicas, 3,(1) 139-156. Recuperado de https://revistaestudiospoliticaspublicas.uchile.cl/index.php/REPP/article/view/46356

Polo, S.D., Hidalgo, R.L., y Jiménez, M. (2010). Impacto del Gobierno Corporativo en Empresas Méxicanas Cotizadas. Código de Mejores Prácticas Corporativas Estudio Empírico (2005-2010). Asociación Nacional de Facultades y Escuelas de Contaduría y Administración. http://congreso.investiga.fca.unam.mx/docs/xviii/docs/5.06.pdf

Polo, S.D., Villegas, E., Jiménez, M. y Martín, D. (2018). Influencia Del Comité De Auditoría En El Desempeño Financiero Organizacional, En Empresas Mexicanas Cotizadas (2010-2014). European Scientific Journal, 14(19), 135-148. https://doi.org/10.19044/esj.2018.v14n19p135

Poveda, F. (2015). Cuestiones Estadísticas Sobre Modelos y Contrastes De Ajustes Por Devengo Anormales. Working Paper, January 2001, 1-52. Recuperado de https://www.researchgate.net/publication/5134718

Reguera, A. N., Laffarga, J., y De Fuentes, P. (2015). Modelos de gestión de resultados: Un estudio transnacional. Revista de Contabilidad, 18(1), 11-19. https://doi.org/10.1016/j.rcsar.2014.01.001

Sáenz, J. y García, E. (2014). Does Corporate Governance Influence Earnings Management in Latin American Markets ? J. Bus Ethics, 121, 419-440. https://doi.org/10.1007/s10551-013-1700-8

Sánchez, I.L. y Campos, E. (2010). Industria manufacturera y crecimiento economic en la frontera norte de México. Región y Sociedad, 22(49), 1870-3925. Recuperado de http://www.scielo.org.mx/scielo.php?script=sci_arttext\&pid=S1870-39252010000300003 
Shah, S. Z. A., Zafar, N., y Durrani, T. K. (2009). Board Composition and Earnings Management an Empirical Evidence Form Pakistani Listed Companies. Middle Eastern Finance and Economics, 3(3), 28-38.

Recuperado de http://citeseerx.ist.psu.edu/viewdoc/download?doi=10.1.1.334.372\&rep=rep1\&type=pdf

Tran, D.H. (2014). Multiple Corporate Governance Attributes and the Cost of Capital evidence From Germany. The British Accounting Review, 10, 179-197. https://doi.org/10.1016/j.bar.2014.02.003

Tejedo, F. y Ferraz, J. (2018). Estrategia de gestión y divulgación del capital intelectual: Influencia del Gobierno Corporativo. Contaduría y Administración, 63(2), 1-18. Recuperado de http://www.scielo.org.mx/scielo.php?script=sci_arttext\&pid=S0186-10422018000200011

Varshney, P., Kumar, V. y Vasal, V. K. (2012). Corporate Governance Index and Firm Performance: Empirical evidence from India. Electronic copy available, 1-35. http://dx.doi.org/10.2139/ssrn.2103462

Vihi, S. K., Abu, A., y Lortima T. (2019). Some Aspects of Corporate Governance Mechanisms and Earnings Management: Evidence from Two Critical Sectors of Nigerian Stock Exchange. IOSR Journal of Business and Management (IOSR-JBM), 21(2), 10-17. http://www.iosrjournals.org/iosr-jbm/papers/Vol21-issue2/Series 1/B2102011017.pdf

Villeda, L. (2015). Gobierno Corporativo, Mecanismo para Generar Esquemas de Competitividad e Innovación en las Pyme concentradas en Santiago de Cali: Un Estudio De Casos. (Tesis de maestría) Universidad Autónoma de Occidente, Cartagena, Murcia, España. Recuperado de https://red.uao.edu.co/bitstream/10614/7028/1/T05111.pdf

Wanjohi, G. (2013). The relationship between corporate governance practices and Earnings management for companies quoted at the Nairobi securities exchange. ( Master's Thesis) University of Nairobi.Nairobi, Kenia. Recuperado de http://erepository.uonbi.ac.ke/bitstream/handle/11295/58619/Muchoki_The\%20Relationship\%20betwee n\%20Corporate\%20Governance\%20Practices\%20and\%20Earnings\%20Management\%20for\%20Companies \%20.pdf?sequence $=3$

Esta obra está bajo una Licencia Creative Commons Attribución-NoCommercial 4.0 International

\section{(c) EYY-NC}

